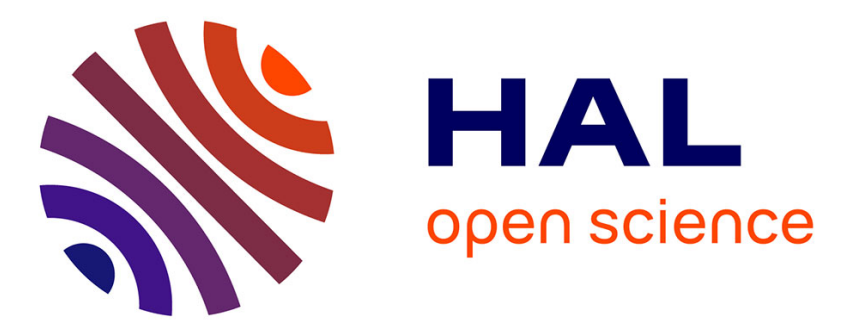

\title{
Vers une production en temps réel d'intervalles prédictifs associés aux prévisions de crue dans Vigicrues en France
}

Julie Viatgé, Lionel Berthet, Renaud Marty, François Bourgin, Olivier Piotte, Maria-Helena Ramos, Charles Perrin

\section{To cite this version:}

Julie Viatgé, Lionel Berthet, Renaud Marty, François Bourgin, Olivier Piotte, et al.. Vers une production en temps réel d'intervalles prédictifs associés aux prévisions de crue dans Vigicrues en France. La Houille Blanche - Revue internationale de l'eau, 2019, 2, pp.63-71. 10.1051/hb/2019016 . hal02610239

\section{HAL Id: hal-02610239 \\ https://hal.inrae.fr/hal-02610239}

Submitted on 16 May 2020

HAL is a multi-disciplinary open access archive for the deposit and dissemination of scientific research documents, whether they are published or not. The documents may come from teaching and research institutions in France or abroad, or from public or private research centers.
L'archive ouverte pluridisciplinaire HAL, est destinée au dépôt et à la diffusion de documents scientifiques de niveau recherche, publiés ou non, émanant des établissements d'enseignement et de recherche français ou étrangers, des laboratoires publics ou privés. 


\title{
Vers une production en temps réel d'intervalles prédictifs associés aux prévisions de crue dans Vigicrues en France
}

\author{
Julie Viatgé 1,*$^{*}$ Lionel Berthet ${ }^{2}$, Renaud Marty $^{2}$, François Bourgin ${ }^{3}$, Olivier Piotte ${ }^{4}$, \\ Maria-Helena Ramos ${ }^{1}$ et Charles Perrin ${ }^{1}$ \\ 1 Irstea, UR HYCAR, 1 rue Pierre-Gilles de Gennes, CS 10030, 92761 Antony Cedex, France \\ 2 DREAL Centre-Val-de-Loire, 5, avenue Buffon, CS 96407, 45064 Orléans, France \\ 3 IFSTTAR, Allée des Ponts et Chaussées, CS 5004, 44344 Bouguenais, France \\ 4 SCHAPI, 42, avenue Gaspard Coriolis, 31057 Toulouse, France
}

Reçu le 16 février 2018 / Accepté le 8 janvier 2019

\begin{abstract}
Résumé - La quantification des incertitudes en modélisation est un sujet ancien, mais de nombreux développements ont été réalisés au cours des quinze dernières années dans un contexte de prévision des crues, avec l'essor notamment des approches probabilistes. En France, au sein du réseau national de prévision des crues (Vigicrues), cela a conduit à la mise au point d'une méthode automatisée de quantification des incertitudes prédictives (OTAMIN) rentrant dans la catégorie des approches de \posttraitement, qui génère des intervalles de confiance sur les prévisions de débit, et d'un outil d'expertise par les prévisionnistes de ces intervalles (EAO/EXPRESSO). Cet article présente les principes méthodologiques de cette démarche, en donne une illustration d'application opérationnelle récente et discute de son utilité pour des utilisateurs institutionnels et pour le grand public. Quelques perspectives d'amélioration sont avancées.

Mots clés : incertitudes / prévision des crues / probabilité / fiabilité / OTAMIN / EAO / EXPRESSO

Abstract - Towards the real-time production of predictive intervals around streamflow forecasts in Vigicrues in France. Although quantifying uncertainties in modelling is an old topic, many developments have been made over the past fifteen years in the context of flood forecasting, mainly with the development of probabilistic approaches. In France, within the national network for flood forecasting (Vigicrues), developments have been made to propose a post-processing methodology to quantify predictive uncertainty (OTAMIN), which automatically generates confidence intervals around streamflow forecasts, and a tool that is used by forecasters to expertise these confidence intervals (EAO/EXPRESSO). This paper presents the main principles of this general approach, gives an example of its recent application in operational conditions and discusses its usefulness for the users and the general public. Some perspectives of improvement are discussed.
\end{abstract}

Keywords: uncertainties / flood forecasting / probability / reliability / OTAMIN / EAO / EXPRESSO

\section{Introduction}

\subsection{Système de prévision et incertitudes}

Les systèmes de prévision des crues et des inondations reposent sur trois piliers communs (Ramos, 2018) :

- les données, qu'elles soient historiques, observées en temps réel ou prévues (pour les entrées météorologiques);

- les modèles, qu'ils soient hydrologiques ou hydrauliques ;

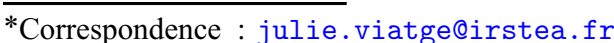

- les prévisionnistes, en charge de la mise en œuvre des systèmes et de l'interprétation de sorties brutes de modèles multiples, tout en faisant face aux contraintes organisationnelles de prévision en temps réel.

Ces trois composantes introduisent des incertitudes chacune à leur niveau, et influent sur le flux d'expertise et de décisions inhérent à ces systèmes, allant de la prévision, l'alerte météorologique et hydrologique, à la cartographie des zones potentiellement inondables (Ramos et al., 2010 ; Berthet et al., 2016). De ce fait, les décisions basées sur les prévisions, par ex. la mise en alerte de services de secours, l'évacuation de 
personnes ou les manœuvres d'ouvrages, sont prises en contexte souvent fortement incertain, et l'estimation des incertitudes associées aux prévisions à diverses échéances revêt donc toute son importance.

\subsection{Prise en compte des incertitudes dans la chaîne de prévision opérationnelle}

Les sorties de modèles de prévision des crues fournies en temps réel sont affectées par les incertitudes liées (1) aux observations rapatriées en temps réel (pluie, débit, température, etc.), (2) aux conditions météorologiques futures (notamment les précipitations) et (3) aux modélisations utilisées (structure, paramétrage, résolution numérique, résolutions spatiales et temporelles). La prise en compte de ces principales sources d'incertitude, qui peuvent être caractérisées de manière indépendante l'une par rapport à l'autre (Krzysztofowicz, 2002), a un impact important sur la nature et la qualité des prévisions de débit ou hauteur d'eau produites par les systèmes de prévision, et par voie de conséquence, sur l'interprétation qui en est faite par le prévisionniste et sur les mesures prises ensuite dans la chaîne de décision et d'alerte.

Bien que l'importance des incertitudes ait été reconnue depuis de nombreuses années (voir par. ex. Krzysztofowicz, 1999), leur prise en compte explicite dans les systèmes de prévision n'est pas encore une pratique courante (Ramos, 2018). Ceci peut être expliqué, d'une part, par la difficulté à évaluer explicitement les erreurs de modélisation et les incertitudes des observations hydrométéorologiques, et, d'autre part, par le manque de pratique dans l'évaluation de prévisions hydrologiques incertaines (Franz et Hogue, 2011).

Une approche aujourd'hui très courante pour tenir compte et quantifier les incertitudes est la prévision d'ensemble. Le principe est de dépasser les limites des approches déterministes en considérant des ensembles d'options possibles (par ex. modèles ou jeux de paramètres, ou scénarios de prévisions météorologiques) (voir Cloke et Pappenberger, 2009 et les travaux du groupe HEPEX, www.hepex.org). Dans ce cadre, différentes approches existent, allant de la prise en compte des incertitudes de chaque composante du système de prévision à l'utilisation d'approches qui permettent de quantifier de manière globale et agrégée l'incertitude prédictive totale.

D'une part, les méthodes d'assimilation de données (par ex., filtre de Kalman ou filtre particulaire) peuvent notamment être utilisées pour représenter de manière probabiliste et mettre à jour les états et les paramètres d'un ou plusieurs modèles hydrologiques ou hydrauliques (voir Berthet, 2010 ; Liu et al., 2012). D'autre part, les méthodes de post-traitement statistique permettent quant à elles de caractériser les distributions de probabilité conditionnelles des variables prédites à partir d'une période d'apprentissage et d'approches statistiques adaptées. Le but est de quantifier l'incertitude prédictive totale et rendre les prévisions justes et fiables, c'est-à-dire, avec des probabilités calibrées statistiquement, pour la prise de décision.

Les premières études proposant des post-traitements statistiques de prévisions hydrologiques à courte échéance ont porté sur des approches basées sur le formalisme bayésien, combinées ou non à une approche de prévision météorologique d'ensemble pour quantifier l'incertitude des forçages (Krzysztofowicz, 2001; Reggiani et Weerts, 2008). D'autres développements et applications ont vu le jour, notamment basés sur des approches non paramétriques, à noyaux, basées sur des copules ou la transformation en ondelettes (par ex. Madadgar et Moradkhani, 2012), les erreurs de calage du modèle hydrologique (Olsson et Lindström, 2008), la prise en compte du lien probabiliste entre l'erreur de prévision et des variables explicatives liées au processus de prévision hydrologique (Montanari et Grossi, 2008), ou l'utilisation d'une approche bayésienne avec une copule empirique pour la prise en compte des dépendances spatio-temporelles des prévisions (Bellier et al., 2018). Une revue détaillée de la littérature a récemment été proposée par Li et al. (2017).

À Irstea, les travaux de thèse de Bourgin (2014) ont abouti à une méthode de post-traitement, permettant de caractériser de manière empirique, non paramétrique, la distribution des erreurs multiplicatives (ou additives) selon la gamme de débits (ou hauteurs d'eau) simulés par le modèle hydrologique et l'horizon de prévision. Utilisée en opérationnel, elle permet d'associer de manière automatique et systématique des intervalles de confiance aux prévisions hydrologiques ou hydrauliques obtenues avec différents modèles déterministes.

\subsection{Evolution de la prise en compte des incertitudes au sein du réseau Vigicrues}

Depuis la réforme de la prévision des crues en France en 2003, le réseau national de la prévision, composé des Services de prévision des crues (SPC) et du Service central d'hydrométéorologie et d'aide à la prévision des inondations (SCHAPI), et désormais appelé Vigicrues, a progressivement amélioré ses capacités de prévision, en intégrant des outils de modélisation dédiés et de plus en plus de sources d'information. Les incertitudes ont été d'abord prises en compte de manière simple, par des fourchettes de scénarios météorologiques considérés et dans la dispersion des abaques de prévision hydrologique utilisés, avec des estimations «à dire d'expert» et une communication qualitative textuelle. Vers 2010, la quantification des incertitudes est devenue un sujet incontournable au sein du réseau: l'identification du besoin d'afficher, à terme, des prévisions graphiques sur Vigicrues s'est accompagnée du besoin de les assortir d'incertitudes prédictives.

Après des journées techniques fin 2011, deux groupes de travail constitués respectivement de membres du réseau Vigicrues et d'experts scientifiques ont mené entre 2012 et 2014 des travaux visant à bâtir un socle commun sur la question de l'estimation et de la communication des incertitudes prédictives (Berthet et al., 2016). Cette phase exploratoire a abouti en 2014 à une feuille de route intégrant notamment le développement en 2015 d'outils à visée opérationnelle : un outil d'estimation automatique et calibrée des incertitudes de modélisation, réalisé par Irstea, et un outil d'expertise «finale» pour le prévisionniste, développé en interne par le réseau Vigicrues. Après une dernière ligne droite consacrée à la préparation technique et organisationnelle pour le passage à l'opérationnel, ainsi qu'à des expérimentations menées avec les SPC, la production et l'affichage de prévisions graphiques accompagnées d'incertitudes sur le site Vigicrues de diffusion des prévisions (www.vigicrues.gouv.fr) sont effectifs depuis octobre 2017. Le nombre de points de prévision concernés s'étend progressivement: de 74 stations à l'automne 2017, il est passé à 129 stations au printemps 2018. 

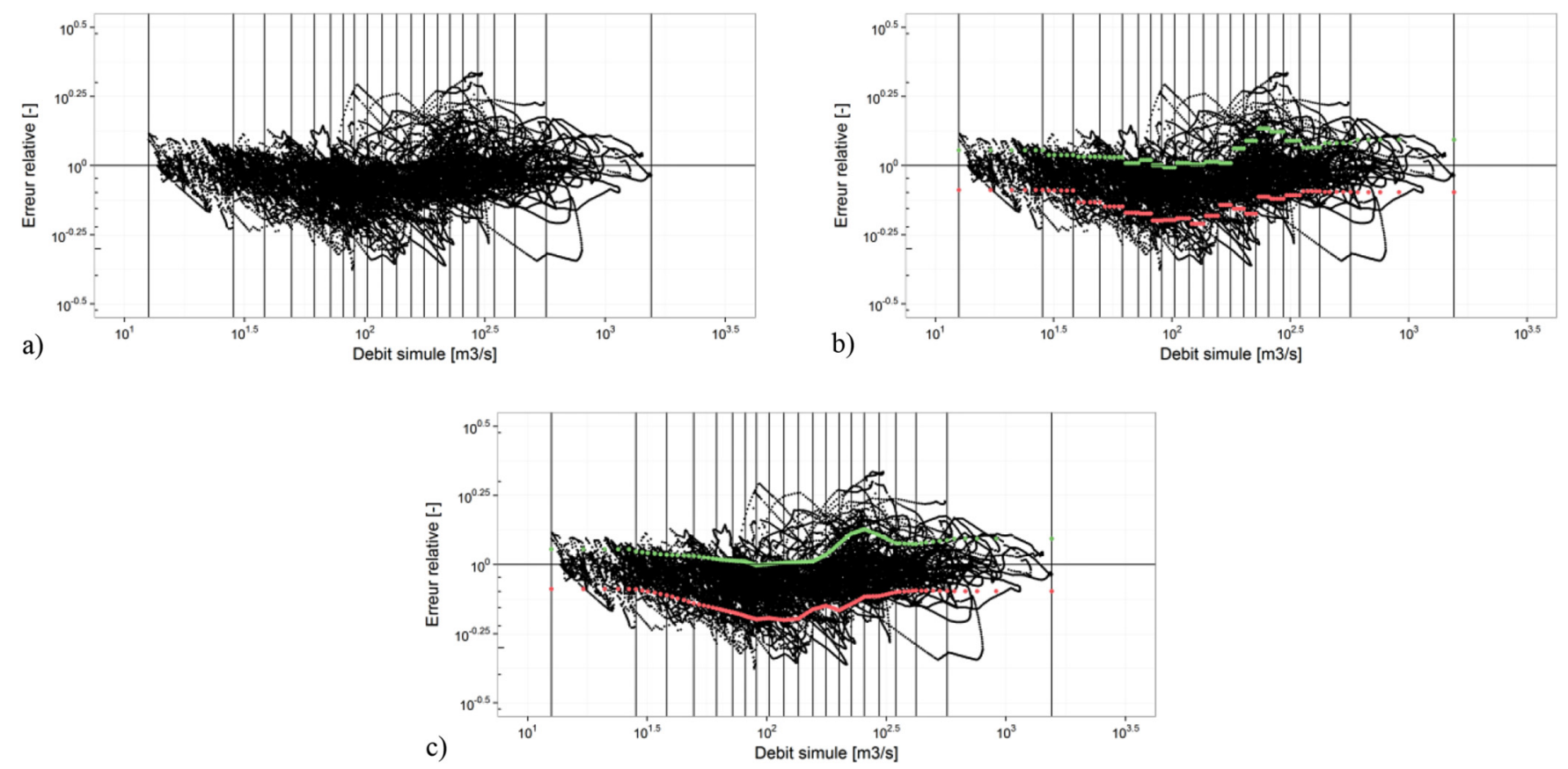

Fig. 1. Étapes de la méthode QUOIQUE appliquée à des erreurs relatives de débits. a : Les erreurs relatives sont réparties en 20 classes de débit simulé ; b : Les quantiles d'erreur 10 (en rouge) et 90 (en vert) sont calculés par classe de débit simulé; c: Les quantiles sont lissés par une moyenne mobile sur 101 points de référence.

\subsection{Objectifs de l'étude}

Cet article vise à présenter la démarche de quantification des incertitudes proposée au sein du réseau Vigicrues. Deux outils sont utilisés pour estimer des intervalles prédictifs (terme entendu au sens large comme un intervalle quantifiant les incertitudes) sur les prévisions en temps réel : OTAMIN, d'une part, permet l'estimation des incertitudes brutes de modèles hydrologiques ou hydrauliques; EAO/EXPRESSO, d'autre part, est un outil qui permet au prévisionniste d'apporter son expertise aux gammes d'erreurs proposées. Ces deux outils sont présentés, ainsi qu'un retour d'expériences sur leur utilisation au sein du SPC Loire-Cher-Indre. Enfin, les limites et perspectives d'amélioration de l'approche proposée sont discutées.

\section{Description de la méthode}

\subsection{Description de la méthode QUOIQUE et du produit OTAMIN}

Le logiciel OTAMIN (OuTil Automatique d'estiMation des INcertitudes prédictives) est un produit d'estimation des incertitudes applicable à des modèles de prévision hydrologiques ou hydrauliques. Cet outil permet notamment d'associer en temps réel des intervalles de confiance à des prévisions déterministes, intervalles qui peuvent être ensuite expertisés par les prévisionnistes.

L'outil opérationnel est composé de trois utilitaires:

- l'utilitaire d'analyse des séries passées permet de juger de la fiabilité de la méthode par une analyse en calage-validation;

- l'utilitaire de calage s'utilise ensuite, et permet de caler la méthode en déterminant des intervalles de confiance en fonction de différentes gammes de valeur (débit ou hauteur d'eau) simulée ;
- l'utilitaire temps réel utilise les statistiques calculées par l'utilitaire de calage et les applique aux prévisions déterministes fournies.

Pour réaliser ces estimations d'incertitudes, OTAMIN exploite en particulier la méthode QUOIQUE (QUantification Opérationnelle des Incertitudes par QUantiles de dEbit) développée par Bourgin (2014). Cette méthode repose sur l'analyse a posteriori des erreurs passées du modèle calculées sur une période conséquente (au moins 1000 couples de valeurs observées et prévues sont nécessaires pour appliquer la méthode), afin de permettre une analyse statistique fiable.

La méthode QUOIQUE comprend plusieurs étapes successives (voir Fig. 1):

- les erreurs relatives ou additives du modèle sont calculées et classées en fonction de la valeur prévue. Afin de prendre en compte les différences de structure des erreurs entre les valeurs faibles et fortes, on découpe l'ensemble des valeurs en 20 groupes de même taille (voir Fig. 1a, les barres verticales correspondent aux limites des différents groupes);

- pour chacun de ces 20 groupes de valeurs simulées, des quantiles d'erreur sont calculés (les valeurs de quantiles souhaités sont paramétrables). Sur la figure $1 \mathrm{~b}$, sont tracés les quantiles $10 \%$ (en rouge) et $90 \%$ (en vert) des distributions pour chaque intervalle considéré;

- un lissage est ensuite effectué, en calculant une moyenne mobile sur 10 points voisins pour 101 points de référence qui sont, par défaut, les valeurs minimales et maximales simulées ainsi que les 99 centiles (voir Fig. 1c). 
Ces quantiles d'erreurs sont ensuite regroupés dans des abaques dans lesquels sont renseignées, pour chaque horizon de prévision et pour chaque point de référence, les valeurs de quantile d'erreurs associées. Ces abaques seront ensuite utilisés par l'utilitaire temps réel d'OTAMIN pour fournir des intervalles de confiance aux prévisions déterministes. Concrètement, en temps réel, si les quantiles d'erreurs calculés sont les quantiles 10 et $90 \%$, les abaques fournissent, pour toute une gamme de valeurs prévues, des intervalles de confiance à $80 \%$. Pour des valeurs prévues qui sortent de la gamme observée, les intervalles les plus proches sont extrapolés linéairement.

\subsection{De l'incertitude brute à l'incertitude expertisée}

Les intervalles de confiance fournis par OTAMIN peuvent ensuite être expertisés par les prévisionnistes, avec un outil dédié, développé au sein du réseau Vigicrues (EAO / EXPRESSO). L'outil leur permet de modifier graphiquement tout ou partie des prévisions représentées sous la forme de trois tendances. Au départ, le prévisionniste compare l'ensemble des sorties brutes disponibles (différents modèles ou différents scénarios) et en sélectionne une pour démarrer son expertise. Chaque tendance est initialisée par une série de quantiles de débit (ou de hauteur d'eau) en fonction du temps, estimée par OTAMIN. Ainsi, par défaut, la tendance basse initiale est la série estimée par OTAMIN du quantile associé à la probabilité 0,1 en fonction du temps, la tendance centrale, celle du quantile associé à la probabilité 0,5 , et la tendance haute, celle du quantile associé à la probabilité 0,9 . Le prévisionniste peut modifier "en bloc» une partie d'une ou plusieurs tendances simultanément afin d'augmenter ou de réduire une pointe de crue, de ralentir ou d'accélérer une montée ou une décrue.

Pour guider son analyse, le prévisionniste peut afficher les prévisions précédemment validées (pour des cycles de prévision récents), ou bien la prévision en cours d'expertise sur un autre point de prévision, ou encore un «proxy» basé sur la combinaison linéaire de prévisions en cours d'expertise sur un ou plusieurs autres points (amont ou aval), ou enfin la prévision actuellement disponible sur Vigicrues. Il peut également choisir de repartir d'une telle série pour démarrer son expertise. Ces outils aident les prévisionnistes à conserver la cohérence spatio-temporelle des prévisions et de leurs incertitudes sur le linéaire des cours d'eau (Berthet et al., 2018).

Enfin, des outils complémentaires permettent de «mettre en forme» la prévision pour l'affichage sur Vigicrues. Cela peut se faire en réinterpolant la série (sur des pas de temps réguliers), en sélectionnant certains points seulement (par ex., les pointes), en lissant une partie des tendances, en recollant la prévision à la dernière observation, etc. Les prévisionnistes peuvent également combiner «à la main» différentes prévisions brutes.

\section{Evaluation de la méthode}

\subsection{Test sur un large échantillon de bassins}

L'utilitaire OTAMIN d'analyse des séries passées permet d'évaluer la fiabilité de la méthode sur le jeu de données fourni, par une procédure de calage-validation. Le principe est le même que dans le cas de validations croisées employées sur des modèles hydrologiques : la chronique étudiée est séparée en deux périodes test de même taille; la méthode OTAMIN est calée sur la première période $\mathrm{P} 1$ pour déterminer les gammes d'incertitude; une analyse du pourcentage de données qui rentrent dans ces gammes sur la seconde période P2 permet d'évaluer si le calage de la méthode est fiable sur une période inconnue (c'est-à-dire, que l'intervalle de confiance à $X \%$ contient bien $X \%$ des observations). On fait ensuite de même sur P1, après un calage de la méthode sur P2. Il s'agit d'une procédure d'évaluation plus exigeante qu'une procédure de «leave-one-out» souvent utilisée. Sur la figure 4 est tracé un exemple de sortie: pour chaque quantile d'erreur calculé sur une période, on trace sa fréquence de non-dépassement sur l'autre période. Les points P11 (en gris) et P22 (en bleu), qui correspondent au calage et à la validation sur la même période, sont nécessairement sur la bissectrice; les points P12 (en rouge) correspondent à la fréquence de non-dépassement sur la période 2 après calage sur la période 1, et vice versa pour les points P21 (en vert). Compte tenu de la variabilité hydrologique, les erreurs en validation sont différentes de celles du calage et un écart des points P12 et P21 à la bissectrice n'est donc pas anormal. Juger de l'acceptabilité de cet écart, et ainsi de la validité de la méthode, peut s'avérer être un exercice difficile. Par exemple, sur la figure 4 à gauche, pour le quantile $20 \%$ déterminé sur la période P1 (point rouge à 20), le pourcentage de non-dépassement sur la période $\mathrm{P} 2 \mathrm{n}$ 'est pas de $20 \%$ mais de $15 \%$. Cela indique une légère sous-estimation du quantile d'erreur $20 \%$ sur la période $\mathrm{P} 1$ par rapport à la période P2.

Une analyse de la distribution de ces écarts sur un échantillon de 202 bassins versants en France métropolitaine (Fig. 2a) a été réalisée afin de fournir des gammes d'écarts de référence (Viatgé et al., 2017). La méthode QUOIQUE a été appliquée sur les erreurs relatives obtenues avec le modèle de prévision GRP (Tangara, 2005; Berthet, 2010), sur des chroniques continues de 10 ans (01/01/1997 à 31/12/2006), au pas de temps horaire. Le modèle GRP est très couramment utilisé au sein du réseau Vigicrues et a montré son utilité et des limites lors de récents événements hydrologiques majeurs et inhabituels par plusieurs aspects (Peredo et al., 2018). Un quart des modèles GRP opérationnels au sein du réseau Vigicrues sont accompagnés par l'outil OTAMIN pour l'estimation de l'incertitude de modélisation. Le modèle est ici testé avec comme scénario de pluies futures les pluies observées $a$ posteriori, pour limiter les incertitudes liées à la pluie.

La figure 2 montre un exemple de résultats obtenus sur la période $\mathrm{P} 1$ après calage sur $\mathrm{P} 2$ pour les horizons de prévision de $3 \mathrm{~h}$ (Fig. 2b) et $48 \mathrm{~h}$ (Fig. 2c). La distribution des écarts pour un même quantile d'erreur augmente avec l'horizon de prévision: aux faibles horizons de prévision, le modèle a plus de chances de s'écarter toujours de la même façon de l'observation qu'aux horizons plus lointains, où le bénéfice de l'assimilation dans GRP tend à s'estomper (Bourgin et al., 2014).

L'étude a également permis de fournir des tables de références, qui sont un outil de comparaison utile pour l'analyse de l'acceptabilité des écarts observés entre les deux sous-périodes de la procédure de calage-contrôle. L'écart « acceptable » reste toutefois à l'appréciation de l'utilisateur, en 


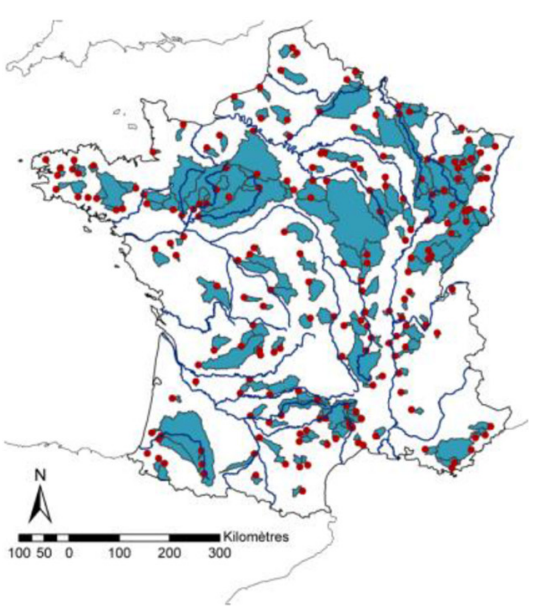

(a)

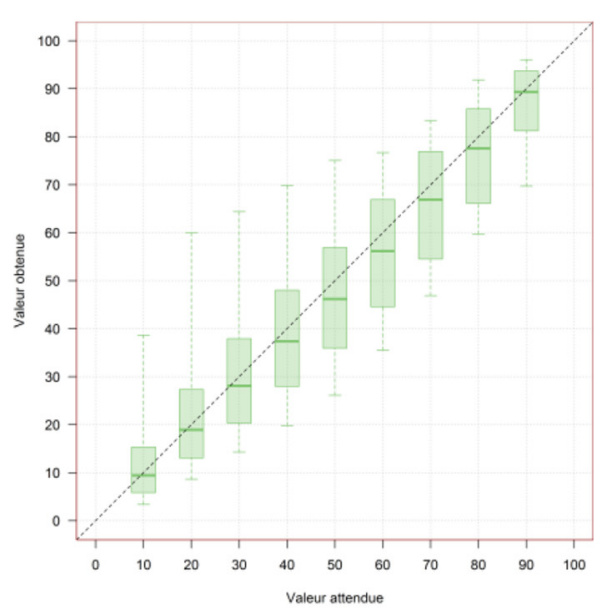

(b)

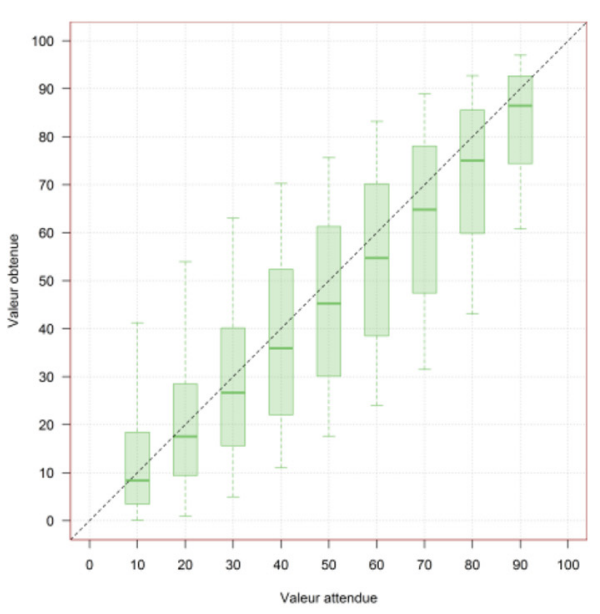

(c)

Fig. 2. a : Contours (en bleu) et exutoires (en rouge) des 202 bassins versants étudiées (Source : Bourgin, 2014); Distribution des écarts à la bissectrice obtenus sur la période P1 après calage sur la période P2 à l'horizon de prévision $3 \mathrm{~h} ; \mathrm{b}: 48 \mathrm{~h}$; $\mathrm{c}$ : sur l'échantillon de 202 bassins versants (Source: Viatgé et al., 2017).
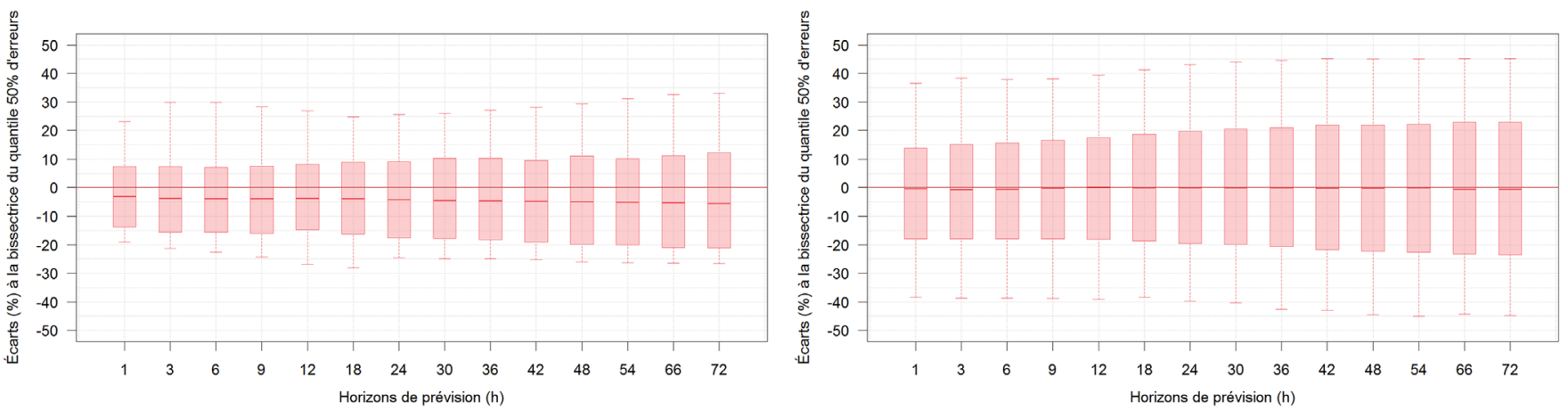

Fig. 3. Distributions des écarts à la bissectrice du quantile $50 \%$ d'erreurs. Ces écarts sont obtenus pour différents horizons de prévision $(1,3,6,9,12$, $18,24,30,36,42,48,54,66$ et 72 h), déterminées sur une chronique longue de 10 ans (gauche) et 2 ans (droite) sur la période de calage-validation P12.

fonction de sa perception des enjeux locaux de la prévision, sachant que le cadre d'évaluation choisi ici est exigeant pour la procédure.

Le nombre de données utilisées étant une condition nécessaire à la fiabilité des résultats, leur sensibilité à la longueur des chroniques a également été étudiée. Les chroniques utilisées ont été divisées par 2 et 5 . La figure 3 montre les distributions des écarts à la bissectrice (sur la période $\mathrm{P} 2$ après calage sur la période $\mathrm{P} 1$ ) pour le quantile d'erreur $50 \%$ à différents horizons de prévision. Les écarts ont été calculés sur des chroniques longues de 10 ans (à gauche) et de 2 ans (à droite). Il apparaît que les écarts à la bissectrice sont accentués avec la perte d'information entraînée par la réduction du nombre de données.

\subsection{Cas d'études opérationnels}

L'application temps réel de l'outil opérationnel OTAMIN est illustrée au travers de prévisions opérationnelles fournies par le modèle hydrologique GRP au cours de l'événement de janvier 2018 sur des affluents bourguignons de la Loire: l'Arconce à
Montceau l'Étoile $\left(600 \mathrm{~km}^{2}\right.$, temps de réponse estimé à $36 \mathrm{~h}$, code K1173210) et la Bourbince à Ciry-le-Noble $\left(336 \mathrm{~km}^{2}\right.$, temps de réponse estimé à 12 h, code K1363010). Ce secteur a été concerné par plusieurs épisodes pluvieux début janvier, engendrant des crues modérées de ces cours d'eau (temps de retour 2 à 5 ans pour l'Arconce et 5 à 10 ans pour la Bourbince).

Dans le cas des modèles GRP opérationnels, des archives de prévision de débit réalisées à partir des entrées de pluies connues (observations de pluie), pour des horizons de prévision de 1 à $72 \mathrm{~h}$, sont disponibles. Ces données sont utilisées pour le calage du modèle à différents horizons de prévision. Les abaques OTAMIN peuvent ainsi être construits à partir de ces données de calage. Sur les bassins étudiés, le calage a été fait sur la période allant d'août 2003 à décembre 2016 et il a été choisi de caler l'abaque de chaque bassin en conservant uniquement les débits supérieurs au débit médian $\left(3,7 \mathrm{~m}^{3} / \mathrm{s}\right.$ à Montceau l'Étoile et $1,8 \mathrm{~m}^{3} / \mathrm{s}$ à Ciry-le-Noble). Cette option proposée par le logiciel OTAMIN permet d'obtenir un échantillonnage plus fin vers les plus fortes valeurs de débit.

Dans un premier temps, les données disponibles pour le calage sont scindées en deux sous-échantillons et une procédure de calage-contrôle est mise en œuvre pour vérifier 

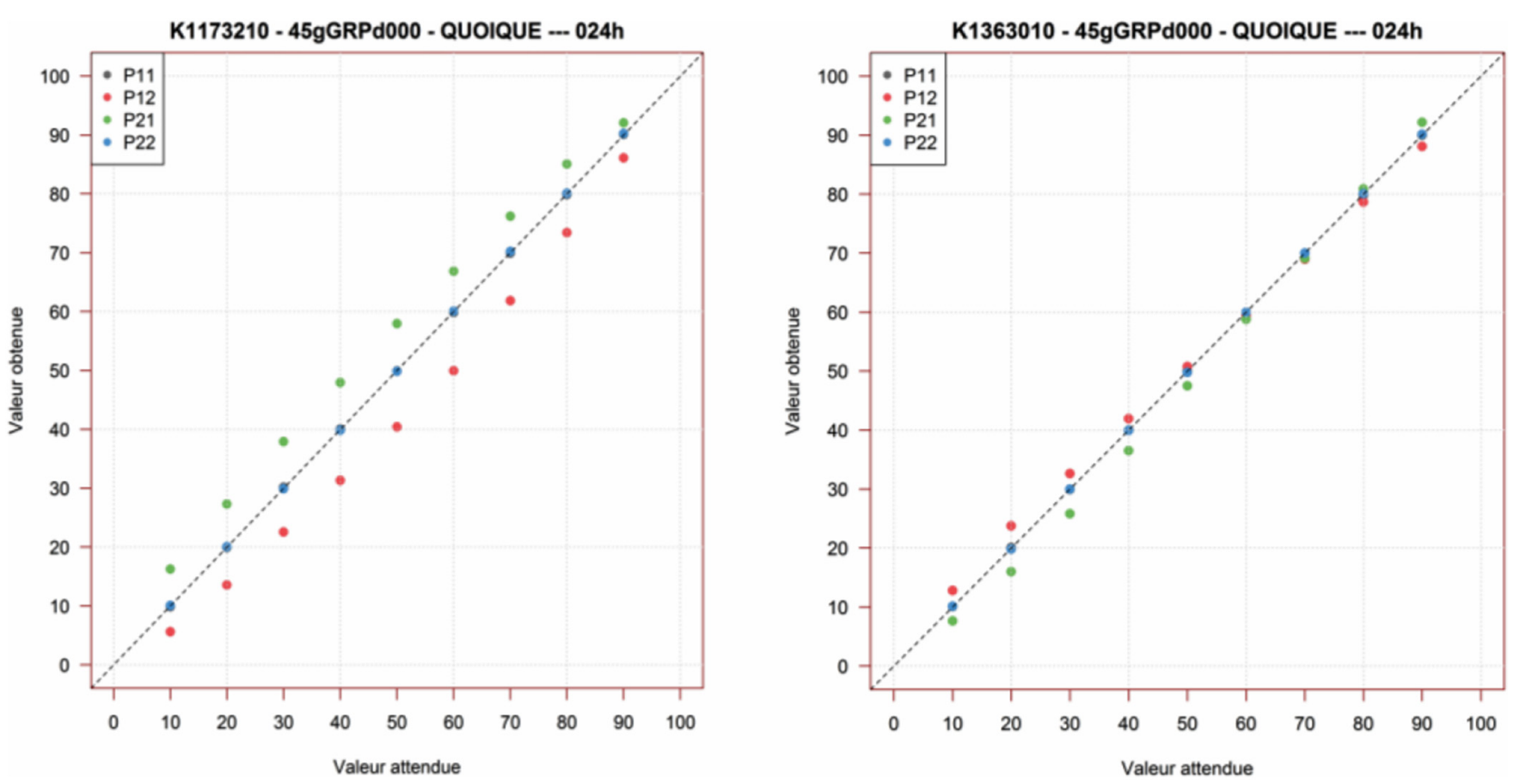

Fig. 4. Comparaison des fréquences d'occurrence des quantiles de débits en calage-contrôle des données de GRP. Les résultats sont présentés sur les modèles à Montceau l'Étoile (gauche) et à Ciry-le-Noble (droite) pour l'horizon de prévision de $24 \mathrm{~h}$. Pxy signifie que l'abaque est calé sur la période $\mathrm{x}$ et vérifié sur la période $\mathrm{y}$.
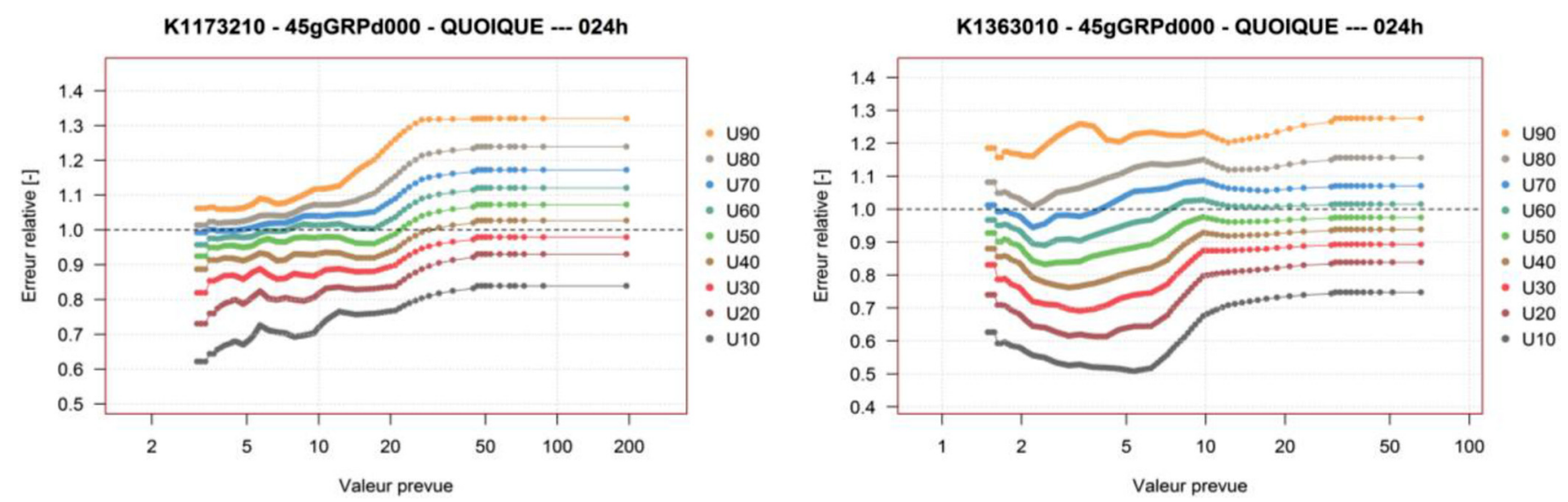

Fig. 5. Erreurs relatives des modèles à Montceau l'Étoile (gauche) et à Ciry-le-Noble (droite) pour l'horizon de prévision de $24 \mathrm{~h}$ (Uxx représente le quantile au non dépassement à $\mathrm{xx} \%$ ).

la robustesse des estimations d'incertitude. La méthode QUOIQUE s'est montrée robuste pour le modèle à Ciry-leNoble: les fréquences d'occurrence théoriques et observées sont très proches sur l'ensemble de la distribution (Fig. 4, droite). La robustesse est moindre mais demeure satisfaisante à Montceau l'Étoile (Fig. 4, gauche). À l'issue de cette phase d'analyse, l'abaque de chaque station est calé sur l'ensemble des données disponibles. La figure 5 illustre les abaques obtenus à l'horizon $24 \mathrm{~h}$ pour les deux bassins d'étude. Par exemple, si le débit (scénario unique) prévu par le modèle GRP de Montceau l'Étoile, à l'horizon de $24 \mathrm{~h}$, est de $50 \mathrm{~m}^{3} / \mathrm{s}$, les valeurs des tendances basse $(10 \%)$, centrale $(50 \%)$ et haute $(90 \%)$ seront, respectivement, de 42,54 et $66 \mathrm{~m}^{3} / \mathrm{s}$.
L'abaque construit lors du calage d'OTAMIN sur la période complète est ensuite appliqué sur les prévisions déterministes issues des modèles opérationnels afin d'estimer l'incertitude liée à la modélisation. Les prévisions déterministes assorties de leur estimation de l'incertitude de modélisation sont ensuite expertisées avant d'être diffusées sur Vigicrues et d'alimenter les modèles hydrauliques en aval. Cette utilisation en temps réel et l'apport de l'expertise sont illustrés par deux exemples de la crue de janvier 2018 (Fig. 6), décrits brièvement ci-après.

Pour le bassin versant de l'Arconce à Montceau l'Étoile (Fig. 6, à gauche), le 5 janvier 2018, les prévisionnistes ont dû tenir compte de la difficulté connue (et documentée dans le SPC) du modèle GRP à reproduire certaines montées rapides des hydrogrammes, 

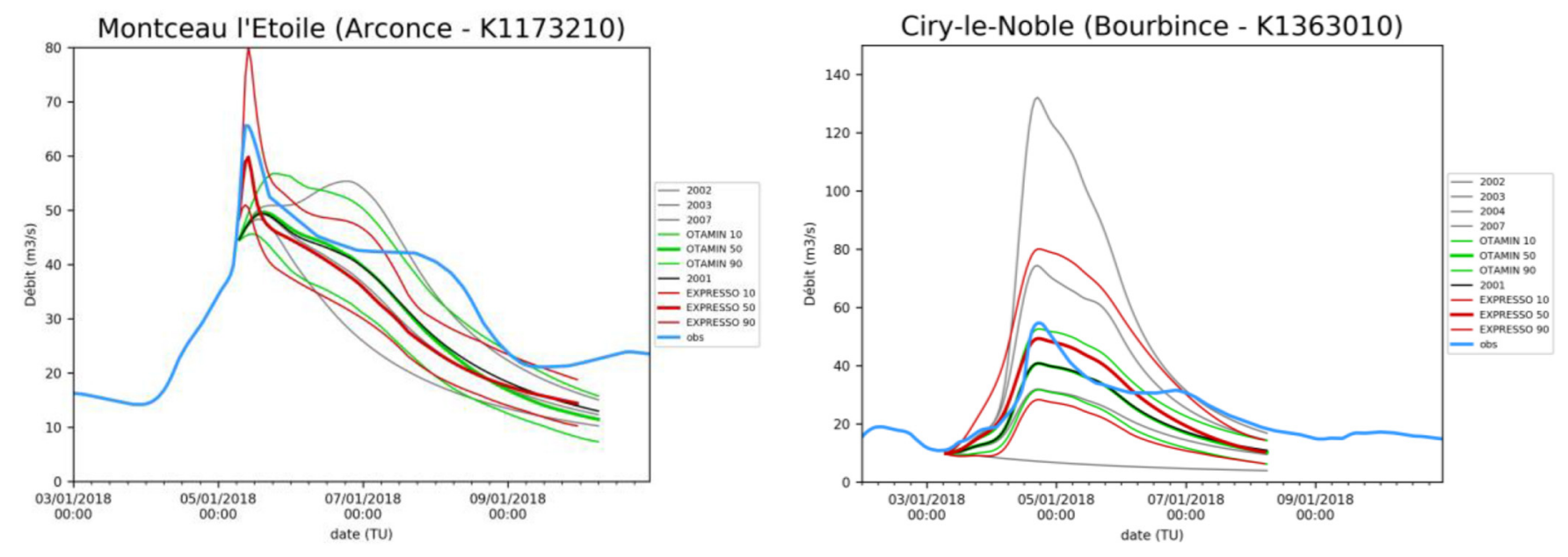

Fig. 6. Prévisions et expertise du 5 janvier 2018 sur le bassin de l'Arconce (gauche) et du 3 janvier 2018 sur le bassin de la Bourbince (droite). Les scénarios bruts sont en gris clair. Le scénario brut support de l'expertise est le 2001. OTAMIN est appliqué à partir de celui-ci (tendances 10, 50 et $90 \%$ en vert). L'expertise est fournie à l'aide de trois tendances construites (courbes rouges) par le prévisionniste dans EXPRESSO.

impliquant un risque de retard et de sous-estimation de la pointe de débit de crue. Il est alors décidé d'augmenter la magnitude des débits de crue modélisée, tout en conservant le gradient de la montée. La tendance centrale est ainsi amplifiée d'environ $20 \%$ sur le pic, comme l'illustre la figure 6 (à gauche, différences entre les courbes OTAMIN et EXPRESSO).

Pour le bassin versant de la Bourbince à Ciry-le-Noble, nous illustrons un autre aspect important de la prévision opérationnelle (Fig. 6, droite). Les outils mis à disposition des prévisionnistes ne permettent pas l'intégration automatique des deux principales sources d'incertitude de la prévision de débit, à savoir l'incertitude météorologique et l'incertitude de la modélisation hydrologique. Les prévisionnistes ont néanmoins la possibilité de les combiner «à la main». Par exemple, le 3 janvier 2018, plusieurs prévisions de débit correspondant à des scénarios de pluie plus ou moins élevés étaient disponibles pour les prévisionnistes à Ciry-le-Noble. L'expertise apportée aux sorties du modèle a ici consisté à élargir le faisceau de prévision constitué par les tendances basse et haute, et à augmenter la valeur de la tendance centrale, tout en s'appuyant sur les scénarios disponibles. Ainsi, l'incertitude prédictive totale a été estimée à partir d'une ébauche fournie par OTAMIN en sortie du modèle GRP et de la diversité des réponses hydrologiques liées aux différents scénarios de conditions météorologiques futures disponibles pour les prévisionnistes. Des applications d'Otamin à des modèles autres qu'hydrologiques existent également (par exemple sur des modèles hydrodynamiques utilisant le code 1D Mascaret; Habert, 2017), mais ne sont pas abordées ici du fait d'une représentativité encore insuffisante.

\section{Discussion et conclusion}

\subsection{Quelle utilité des incertitudes pour la vigilance et la prévision?}

Plusieurs travaux ont été menés pour quantifier l'apport de systèmes de prévision probabiliste dans différents secteurs d'activité (par ex., gestion de réservoirs d'eau ou prévision de crue), suivant des cadres plutôt théoriques ou appliqués à des systèmes de prévision définis (Roulin, 2007 ; Verkade et Werner,
2011 ; Boucher et al., 2012 ; Pappenberger et al., 2015 ; Thiboult et al., 2017). Ramos (2018) discute de la pertinence de mieux évaluer ce lien entre qualité et utilité/valeur des prévisions. Ici, nous donnons une analyse qualitative sur la base de retours opérationnels concernant la mise en place de prévisions avec incertitudes dans le réseau Vigicrues.

Lors de récents retours d'expérience sur des événements de crue vécus par le SPC LCI, ses interlocuteurs en préfecture ont, dans leur grande majorité, fait part de leur satisfaction de disposer de prévisions incluant une information sur l'incertitude. Quelques-uns ont indiqué apprécier la signification précise de chaque tendance, qui les guide dans la prise de décision. D'autres utilisent l'information sur l'incertitude prédictive de façon beaucoup plus qualitative. De façon générale, la grande majorité des plans et des outils d'aide à la décision ne prennent pas encore en compte, de façon explicite, l'incertitude prédictive.

Pour compléter cette vision, on peut revenir sur l'enquête commandée en 2014 par le SCHAPI dans le cadre des réflexions sur l'évolution du portail Vigicrues, réalisée auprès des services techniques de l'État, services de sécurité civile et services techniques de collectivités, ainsi que du grand public dans des territoires concernés par le risque inondation (Comoe et al., 2015). Sur la question des incertitudes, les institutionnels se déclaraient en majorité (environ $70 \%$ des sondés) «parfaitement » ou « assez bien » familiarisés avec la notion d'incertitude de prévision. Sur ce même échantillon, une grande majorité (environ $80 \%$ ) considérait également que le fait de disposer d'incertitudes sur les prévisions ajoute de l'information.

Aujourd'hui, au-delà de quatre jours, les prévisions météorologiques de Météo-France sont assorties d'un indice de confiance, qui permet au prévisionniste de communiquer de manière qualitative sur la fiabilité de sa prévision: plus la prévision est fiable, plus l'indice de confiance qu'il choisira sera élevé ( 1 : confiance très faible, 5 : confiance très élevée). Cet indice de nature qualitative ne permet pas un retour d'expérience affiné. De même, l'hétérogénéité formelle et méthodologique de l'information communiquée traditionnellement sous forme textuelle sur l'incertitude prédictive associée aux prévisions rend difficile les retours d'expérience. 
Concernant les approches plus quantitatives, telle que celle proposée dans l'outil OTAMIN pour la prévision de débit du réseau Vigicrues, il est encore difficile d'avoir des éléments de réponse consistants sur l'utilité pour le grand public des prévisions accompagnées d'une estimation des incertitudes prédictives.

Quant à l'affichage graphique de prévisions en cours de généralisation, plus standardisé, il est encore trop récent, avec seulement quelques points de prévision ayant connu ces derniers mois un événement suffisamment significatif (vigilance jaune, majoritairement), pour que des prévisions soient produites et regardées. Un premier constat peut toutefois être dressé: parmi les messages adressés par les internautes sur Vigicrues début 2018, la quasi-totalité de ceux concernant les prévisions interrogeait sur l'absence de prévisions graphiques dans certains points de prévision et non sur une quelconque difficulté de compréhension.

Un autre élément corroborant: dans les résultats de l'enquête citée précédemment, l'affichage de graphiques de prévisions accompagnées d'une «fourchette de confiance» arrivait en tête des informations prioritaires recherchées par le grand public ( $89 \%$ des 160 personnes sondées). Sur la base de ces quelques éléments, on peut dire que les prévisions hydrologiques, ainsi que leur accompagnement par des incertitudes prédictives, sont plutôt fortement attendues par le grand public.

\subsection{Pistes d'améliorations de l'approche de quantification des incertitudes}

Si l'approche OTAMIN proposée permet de quantifier de manière automatique les incertitudes d'une modélisation globale en cherchant à garantir un niveau de fiabilité maximale aux informations probabilistes fournies, de nombreuses questions se posent encore, notamment en ce qui concerne la variabilité de cette quantification en fonction du contexte de prévision et la fiabilité des estimations dans des contextes d'extrapolation et de prévisions pour des bassins non jaugés. Cette étape d'estimation de l'incertitude de prévision à l'aide de l'approche OTAMIN permet de renseigner sur les incertitudes de la prévision hydrologique, même si elle peut également présenter des limites lors de certains événements, comme cela a été le cas de sousestimations de certains intervalles de confiance lors de la crue de mai-juin 2016 (Peredo et al., 2018).

Une piste d'amélioration de la méthode réside dans une meilleure caractérisation des situations de prévision, en distinguant, par exemple, les montées des descentes de crues lors de l'estimation des quantiles prédictifs ou en introduisant une saisonnalité dans l'estimation. L'enjeu de ces développements est d'améliorer la finesse des prévisions probabilistes tout en garantissant une bonne fiabilité à ces approches qui reposent sur une période d'apprentissage et avec un nombre de données nécessairement limités.

Les situations d'extrapolation nécessitent par ailleurs une attention particulière : comment faire évoluer les intervalles de confiance associés à des prévisions qui dépassent les valeurs contenues dans la période d'apprentissage? Des travaux sont en cours pour déterminer la meilleure stratégie d'extrapolation, suivant les bassins versants et les horizons de prévision, entre stabilisation des intervalles de confiance et évolution suivant un comportement multiplicatif (Berthet et al., 2017).
Par ailleurs, il reste difficile d'estimer de manière fiable les intervalles de confiance associés à des prévisions obtenues pour des bassins non jaugés. Des travaux ont été menés dans un contexte de simulation par Bourgin et al. (2015). Des études complémentaires devront être conduites pour évaluer la pertinence de l'approche proposée pour la prévision des crues, dans la continuité des travaux menés par Randrianasolo et al. (2011).

Une autre limite de la méthode actuellement mise en œuvre par les services opérationnels est due au fait que les outils sont d'abord pensés pour établir une prévision en un point et à un instant donné et ne garantissent pas la cohérence spatiotemporelle des prévisions (Berthet et al., 2018). Par ailleurs, les premiers retours des services opérationnels montrent également que la maîtrise de la part subjective ou expertisée de l'élaboration des prévisions est un enjeu fort.

Enfin, la généralisation prochaine de la mise à disposition des prévisions météorologiques d'ensemble auprès des SPC ouvrira la voie à une quantification automatisée des incertitudes intégrant à la fois les incertitudes liées au modèle et celles liées aux prévisions de pluie. Bien que l'automatisation de certaines tâches devienne inévitable quand il s'agit de mieux quantifier les principales sources d'incertitudes de la prévision hydrologique, les prévisionnistes continueront à jouer un rôle essentiel dans le processus de prévision, du fait de la complexité et de l'hétérogénéité des processus, de la difficulté de prendre en compte certaines influences d'origine anthropique et du caractère nécessairement imparfait des chaînes de prévision.

Remerciements. Les auteurs remercient le SCHAPI pour son soutien financier au développement du produit OTAMIN et aux travaux de thèse du quatrième auteur. Les données hydrologiques et climatiques nécessaires au test du produit ont été fournies respectivement par la banque HYDRO (gérée par le SCHAPI) et par Météo-France. Les auteurs remercient également les SPC qui ont contribué, par leurs retours et leurs suggestions, à faire avancer la réflexion sur le produit OTAMIN et sur la communication des incertitudes. Enfin, les auteurs remercient les relecteurs anonymes de l'article pour leurs suggestions qui ont permis d'améliorer le texte.

\section{Références}

Bellier J, Zin I, Bontron G. 2018. Generating coherent ensemble forecasts after hydrological postprocessing: Adaptations of ECCbased methods. Water Resour Res. doi: 10.1029/2018WR022601.

Berthet L. 2010. Prévision des crues au pas de temps horaire : pour une meilleure assimilation de l'information de débit dans un modèle hydrologique. Thèse de Doctorat, Cemagref (Antony), AgroParisTech, Paris, 603 p., Disponible sur https://webgr.irstea.fr/wpcontent/uploads/2012/07/2010-BERTHET-THESE.pdf.

Berthet L, Piotte O, Gaume E, Marty O, Ardilouze C. 2016. Operational forecast uncertainty assessment for better information to stakeholders and crisis managers. 3rd European Conference on Flood Risk Management, Oct. 2016, Lyon, France.

Berthet L, Marty R, Bourgin F, Viatgé J, Piotte O, Perrin C. 2017. Combining empirical approaches and error modelling to enhance predictive uncertainty estimation in extrapolation for operational flood forecasting. Tests on flood events on the Loire basin, France. Geophys Res Abstr 19, EGU2017-15679, EGU General Assembly 2017.

Berthet L, Valéry A, Garçon R, Marty R, Puygrenier D, Moulin L, Piotte O. 2018. Cohérence des prévisions et place de l'expertise : les 
nouveaux défis pour la prévision des crues. Actes de la Conférence $S H F$ «De la prévision des crues à la gestion de crise», Avignon, 14-16 novembre 2018.

Boucher MA, Tremblay D, Delorme L, Perreault L, Anctil F. 2012. Hydroeconomic assessment of hydrological forecasting systems. $J$ Hydrol 416-417: 133-144.

Bourgin F. 2014. Comment quantifier l'incertitude prédictive en modélisation hydrologique? Travail exploratoire sur un grand échantillon de bassins versants. Thèse de Doctorat, Irstea (Antony), AgroParisTech, Paris, 230 p., Disponible sur https://webgr.irstea.fr/ wp-content/uploads/2014/09/2014_BOURGIN_these.pdf.

Bourgin F, Ramos MH, Thirel G, Andréassian A. 2014. Investigating the interactions between data assimilation and post-processing in hydrological ensemble forecasting. J Hydrol Part D 519: 2775 2784. doi: 10.1016/j.jhydrol.2014.07.054.

Bourgin F, Andréassian V, Perrin C, Oudin L. 2015. Transferring global uncertainty estimates from gauged to ungauged catchments. Hydrol Earth Syst Sci 19: 2535-2546. Available from 10.5194/ hess-19-2535-2015, doi: 10.5194/hess-19-2535-2015.

Cloke HL, Pappenberger F. 2009. Ensemble flood forecasting. A review. J Hydrol 375: 613-626. doi: 10.1016/j.jhy drol.2009.06.005.

Comoe F, Helloco Consultant, Amenis, Midi-Marketing. 2015. Assistance à maîtrise d'ouvrage pour l'évolution de l'information diffusée sur la prévision des crues et la vigilance associée, ainsi que des moyens de diffusion de cette information. Phase 1-Rapport d'étape 1.3-Approfondissements de la connaissance des besoins. Rapport d'études, Service central d'hydrométéorologie et d'appui à la prévision des inondations (SCHAPI), Toulouse, $141 \mathrm{p}$.

Franz KJ, Hogue TS. 2011. Evaluating uncertainty estimates in hydrologic models: Borrowing measures from the forecast verification community. Hydrol Earth Syst Sci 15: 3367-3382.

Habert J. 2017. Retour d'expérience sur l'utilisation d'Otamin: application au modèle hydraulique Seine Bassée avec assimilation de données. Présentation orale au groupe utilisateurs «OTAMIN", Irstea (Antony), 14 septembre 2017.

Krzysztofowicz R. 1999. Bayesian theory of probabilistic forecasting via deterministic hydrologic model. Water Resour Res 35: 27392750. doi: 10.1029/1999wr900099.

Krzysztofowicz R. 2001. The case for probabilistic forecasting in hydrology. J Hydrol 249(1-4): 2-9.

Krzysztofowicz R. 2002. Bayesian system for probabilistic river stage forecasting. J Hydrol 268: 16-40.

Li W, Duan Q, Miao C, Ye A, Gong W, Di Z. 2017. A review on statistical postprocessing methods for hydrometeorological ensemble forecasting. WIRES Water (4): e1246. doi: 10.1002/wat2.1246.

Liu Y, Weerts AH, Clark M, Hendricks Franssen HJ, Kumar S, Moradkhani $\mathrm{H}$, et al. 2012. Advancing data assimilation in operational hydrologic forecasting: Progresses, challenges, and emerging opportunities. Hydrol Earth Syst Sci 16: 3863-3887.
Madadgar SH, Moradkhani DG. 2012. Towards improved postprocessing of hydrologic forecast ensembles. Hydrolog Process 28. doi: 10.1002/hyp.9562.

Montanari A, Grossi G. 2008. Estimating the uncertainty of hydrological forecasts: A statistical approach. Water Resour Res 44: W00B08.

Olsson J, Lindström G. 2008. Evaluation and calibration of operational hydrological ensemble forecasts in Sweden. $J$ Hydrol 350: 14-24.

Pappenberger F, Cloke HL, Parker DJ, Wetterhall F, Richardson DS, Thielen J. 2015. The monetary benefit of early flood warnings in Europe. Environ Sci Policy 51: 278-91.

Peredo D, Viatgé J, Vrignaud C, Riffiod F, Marty R, Berthet L, et al. 2018. Le modèle hydrologique GRP a-t-il été fiable et utile lors des récentes crues de la Seine et de la Loire. Actes de la Conférence SHF «De la prévision des crues à la gestion de crise», Avignon, 14-16 novembre 2018.

Ramos MH. 2018. Qualité et valeur des prévisions hydrologiques d'ensemble. Mémoire d'habilitation à diriger des recherches (dossier scientifique), Irstea (Antony), Sorbonne Université, Paris, 102 p., Disponible sur https://webgr.irstea.fr/wp-content/uploads/ 2018/05/HDR-RAMOS_2018_VPublic.pdf.

Ramos MH, Mathevet T, Thielen J, Pappenberger F. 2010. Communicating uncertainty in hydro-meteorological forecasts: mission impossible? Meteorol Appl 17: 223-235. doi: 10.1002/ met.202.

Randrianasolo A, Ramos MH, Andréassian V. 2011. Hydrological ensemble forecasting at ungauged basins: using neighbour catchments for model setup and updating. Adv Geosci 29: 1-11. doi: 10.5194/adgeo-29-1-2011.

Reggiani P, Weerts AH. 2008. A Bayesian approach to decisionmaking under uncertainty: An application to real-time forecasting in the river Rhine. J Hydrol 356(1-2): 56-69.

Roulin E. 2007. Skill and relative economic value of medium-range hydrological ensemble predictions. Hydrol Earth Syst Sci 11: 725737.

Tangara M. 2005. Nouvelle méthode de prévision de crue utilisant un modèle pluie-débit global. Thèse de Doctorat, Cemagref Antony, EPHE, Paris, $374 \mathrm{p}$.

Thiboult A, Anctil F, Ramos MH. 2017. How does the quantification of uncertainties affect the quality and value of flood early warning systems? J Hydrol 551: 365-373. doi: 10.1016/j.jhy drol.2017.05.014.

Verkade JS, Werner MGF. 2011. Estimating the benefits of single value and probability forecasting for flood warning. Hydrol Earth Syst Sci 15: 3751-3765.

Viatgé J, Perrin C, Marty R. 2017. Analyse complémentaire des résultats de l'utilitaire d'analyse d'OTAMIN. Ordres de grandeurs sur la transférabilité de l'incertitude. Rapport d'étude, Irstea, Antony, $36 \mathrm{p}$.

Citation de l'article : Viatgé J, Berthet L, Marty R, Bourgin F, Piotte O, Ramos M-H, Perrin C. 2019. Vers une production en temps réel d'intervalles prédictifs associés aux prévisions de crue dans Vigicrues en France. La Houille Blanche 2: 63-71. 